\title{
Untersuchung und Klassifikation der Fahreraufmerksamkeit bei längerer partiell automatisierter Fahrt
}

\author{
Alexander Hugenroth ${ }^{1,2}$ (D) - Alexander Warnecke ${ }^{2} \cdot$ Torsten Bertram $^{1}$ \\ Eingegangen: 11. Januar 2021 / Angenommen: 15. November 2021 / Online publiziert: 20. Dezember 2021 \\ (c) Der/die Autor(en) 2021
}

\section{Zusammenfassung}

Um die Unfallzahlen weiter zu senken, schreibt die Europäische Union ab 2030 eine höhere Fahrerüberwachung für neue Fahrzeuge vor. Bislang liegt der Fokus in einem manuell gefahrenen Fahrzeug auf einer Müdigkeitserkennung als Komfortsystem. Jedoch ändern sich die Anforderungen an den Fahrer und dessen Aufgaben bei steigender Automatisierung der Fahraufgabe, sodass auch die Fahrerbeobachtung/-überwachung weiter entwickelt werden sollte. Die vorliegende Arbeit beschäftigt sich mit der Auslegung von Aufmerksamkeitsaufgaben in Simulator-Fahrversuchen und einem ersten Versuch die aufgenommenen Daten von wenigen Probanden automatisch auszuwerten, um die Machbarkeit zu zeigen. Durch die partielle Automatisierung (nach SAE Level 2) der Fahraufgabe bleiben dem Fahrer bei der hier vorgestellten längeren Aufmerksamkeitsaufgabe nur noch schwache und seltene Reize, sodass von einer Vigilanzaufgabe gesprochen werden kann. Zur Messung des Fahrerzustands wird in dieser Machbarkeitsstudie eine Kombination aus verschieden Körperfunktionen durch unterschiedliche Sensoren erfasst, mit einem künstlichen neuronalen Netzwerk fusioniert und verarbeitet. Es werden verschiedene Kombinationen von Sensoren getestet, um herauszufinden, welche Signale für eine Klassifikation des Fahrerzustands ausreichend oder notwendig sind. Dabei wird darauf geachtet, ob diese Signale in Zukunft durch neue Sensortechnologien auch kontaktlos erfasst werden könnten, um eine Entwicklung dieser zu motivieren. Am Ende wird eine Klassifizierung vorgenommen, die zwischen den hier so genannten Zuständen Vigilant (aufmerksam) und Hypovigilant (nicht mehr aufmerksam) unterscheiden kann, sodass eine anschließende Regelung ein solches Signal verwenden kann, um den Fahrer in einen besseren Zustand zu bringen. Zudem kann der Fahrer gewarnt werden, wenn die Aufmerksamkeit nicht zu den Aufgaben passt, die er in der Automatisierungsstufe zu erledigen hat beziehungsweise für die er verantwortlich ist. Dazu könnten Aufgaben zwischen dem Fahrzeug und dem Fahrer dynamisch verteilt werden, also beispielsweise die Führung in Längs- oder Querrichtung wieder als Fahraufgabe dem Fahrer übergeben werden, um ihn aufmerksam zu halten. Durch eine kontaktlose Erfassung der Aufmerksamkeit gibt es zudem einen Komfortgewinn, da zum Beispiel die Hände nicht mehr das Lenkrad festhalten müssen, um dem Fahrzeug seine Aufmerksamkeit zu bestätigen, wie es heute in einigen Fahrzeugen üblich ist.

Alexander Hugenroth

alexander.hugenroth@tu-dortmund.de

1 Lehrstuhl für Regelungssystemtechnik, TU Dortmund,

Dortmund, Deutschland

2 HELLA GmbH \& Co. KGaA, Lippstadt, Deutschland 


\title{
Analysis and classification of driver attention during long-term partially automated driving
}

\begin{abstract}
In order to further reduce the number of accidents, the European Union plans to stipulate a higher level of driver monitoring for new vehicles from 2030 on. Until now, the focus of driver monitoring in a manual driven vehicle has been on fatigue detection as a comfort system. However, the demands on the driver and his tasks are changing with increasing automation, so that driver observation/monitoring should also be further developed. The present work deals with the design of attention tasks in simulator driving tests and a first attempt to automatically evaluate the recorded data from a few subjects to show the feasibility. Due to the partial automation (according to SAE level 2) of the driving task, the driver is left with only weak and rare stimuli for this attention task, so that we can speak of a vigilance task. To measure the driver's state, in this feasibility study, a combination of divers body functions is recorded by different sensors. This signals will be merged and processed with an artificial neural network. Different combinations of sensors are tested to find out which signals are sufficient or necessary for classification. Thereby it is paid attention to whether these signals could also be recorded with non-contact sensors in the future using new technologies. In the end, a classification is formed that can distinguish between what is here called vigilant (alert) and hypovigilant (no longer alert), so that a subsequent control system can use such a signal to get the driver in a better state. In addition, the driver can be warned if the attention does not match the tasks that he has to do in the automation level. This could be achieved by dynamically distributing tasks between the vehicle and the driver. For example, longitudinal or lateral control could again be handed over to the driver as a driving task to keep him attentive. Non-contact attention recording also provides a gain in comfort, since the hands no longer have to hold the steering wheel to confirm the driver's attention, as it is common practice in some vehicles today.
\end{abstract}

\section{Einleitung}

Das automatisierte Fahren entwickelt sich immer weiter, doch wie schnell und ob der Prozess in Stufen oder gleitend abläuft, steht noch nicht genau fest. Sicher ist jedoch, dass sich die Aufgaben des Fahrers und damit verbundene Anforderungen ändern werden. Wie in [14] beschrieben neigen einige Menschen dazu den Systemen zu viel Vertrauen zuzusprechen, sodass sogar Risiken entstehen können. So kann die Reaktion auf wichtige Signale ausbleiben oder ein Fehlverhalten des Systems erst zu spät oder gar nicht mehr festgestellt werden [16]. Es gibt bereits Fahrzeuge, wie in [22] beschrieben, mit automatisierter Querund Längsführung, die sich ohne Überprüfung des Fahrerzustands in vielen Verkehrssituationen über einen langen Zeitraum (einige Stunden) aktivieren lassen, obwohl es sich noch um partielle Automatisierungen (SAE Level 2: Der Fahrer muss die Fahrumgebung und die Fahraufgabe jederzeit überwachen, das Fahrzeug kann sowohl Quer- als auch Längsführung übernehmen) handelt. Um die Unfallzahlen dennoch zu senken, soll unter anderem die Fahrerbeobachtung/-überwachung laut Europäischer Union durch eine Aufmerksamkeits- und Ablenkungserkennung bis 2030 ergänzt werden [4]. Diese Anforderungen orientieren sich allerdings eher an dem manuellen Fahren und sind im Bezug zu der Benutzung eines Mobiltelefons ausgelegt. Es gibt aktuell auch von Automobilherstellern die Ankündigung für eine umfangreichere Fahrerbeobachtung/-überwachung, sodass ein Halten des Lenkrads zur Angabe der Aufmerksamkeit des Fahrers trotz partieller Automatisierung nicht mehr notwendig sein soll [27]. Das Fahrzeug soll dazu die
Blickrichtung des Fahrers überprüfen und so bestimmen, ob dieser noch auf die Straße sieht und damit die automatisierte Fahraufgabe überwacht oder sich einer fahrfremden Tätigkeit widmet. Hierzu wird in [28] ein Ansatz aufgezeigt, indem diese Überprüfung mit einem Kamerasystem umgesetzt wird. Dabei wird angenommen, dass ein Fahrer, der auf die Straße blickt, in jedem Fall aufmerksam ist. Genau an diesem Aspekt soll der Beitrag anknüpfen; indem untersucht wird, wie sich der kognitive Zustand des Fahrers trotz Blick auf die Straße in einer solchen Situation verändert und wie dies messbar gemacht werden kann.

Die Müdigkeitserkennung wird schon länger in Fahrzeugen eingesetzt, so wird dies in [3] für Premium-Fahrzeuge schon im Jahr 2008 angekündigt. In [13] wird der theoretische Hintergrund einer solchen Erkennung über die Fahrereingaben, zum Beispiel am Lenkrad, gezeigt. Damit wird dann eine Warnung an den Fahrer ausgegeben, wenn dieser Anzeichen von Müdigkeit zeigt. Auch in der Forschung wird die Fahrerzustandserkennung im Kontext des automatisierten Fahrens weiter untersucht und diskutiert. So gibt [23] einen Überblick, welche Entwicklungen und Ergebnisse zur Müdigkeit beim manuellen Fahren existieren und welche Sensoren zur Bestimmung des Zustands zum Einsatz kommen. In [8] wird eine Definition von verschiedenen Fahrerzuständen (Wachheit, Aufmerksamkeit, Müdigkeit, Ablenkung, Vigilanz) gegeben und wie diese erfasst werden können. Hierbei ist die Aussage, dass die Vigilanz, die in der vorliegenden Arbeit erfasst werden soll, besonders für die partielle Automatisierung (SAE Level 2) von Interesse ist. Dahingegen wird ebenso beschrieben, dass die Fahreraufmerksamkeit während einer SAE Level 3 Fahrt 
nicht gemessen werden muss, da dem Fahrer fahrfremde Nebentätigkeiten erlaubt sind, welche die volle Aufmerksamkeit des Fahrers in Anspruch nehmen können. Jedoch spielt die Vigilanz immer noch bei der Übergabe zwischen automatisierter Fahrfunktion und manuellem Fahren eine Rolle.

Gegenstand des vorliegenden Beitrags ist die Untersuchung der Fahreraufmerksamkeit bei längerer partiell automatisierter Fahrt. Dabei wird sowohl die psychische Situation, also die Theorie und Definitionen aus der Psychologie, als auch die physiologische Reaktion der Versuchsperson, also die Messbarkeit von größeren Änderungen in kognitiven Prozessen anhand von Körperfunktionen, betrachtet. Die vorliegende Arbeit zeigt eine Studie mit insgesamt neun Versuchspersonen in einem statischen Fahrsimulator, die als Machbarkeitsstudie gesehen wird, um eine spezielle Aufmerksamkeitsaufgabe in die automatisierte Fahrt zu integrieren. Dazu wird zudem eine Datenverarbeitung der aufgenommenen physiologischen Signale vorgestellt. Hierbei wird analysiert, welche Signale für eine Klassifikation sinnvoll sind und auf welche verzichtet werden könnte, um die generelle Machbarkeit der Datenverarbeitung zu zeigen.

Im folgenden Abschnitt wird die psychische Situation beim längeren automatisierten Fahren und physiologische Signale beschrieben. Die durchgeführte Studie, sowie die Zusammenfassung der Befragung der Probanden vor und nach dem Versuch wird in Abschn. 3 näher erläutert. Die nächsten zwei Abschnitte sind bereits in gekürzter Form in dem Tagungsband (VDI Mechatronik Tagung 2021) veröffentlicht, jedoch hier noch etwas genauer ausgeführt. Abschn. 4 legt die Messmethoden und die Datengenerierung während des Versuchs näher. Die Auswertung der aufgenommenen Daten wird in Abschn. 5 mit der Strukturbeschreibung der neuronalen Netzwerke, der Datenvorbereitung, dem Training sowie den Ergebnissen der Klassifizierung zusammengefasst. Abschließend bietet Abschn. 6 eine kontroverse Einordnung der gezeigten Methoden sowie einen Ausblick für folgende Arbeiten sowohl mit Blick auf die Vigilanz-Studie als auch auf die Datenauswertung.

\section{Psychologie und Physiologie bei längerem automatisierten Fahren}

Die Fahrt in einem automatisierten Fahrzeug wird zu neuen psychologischen Situationen und Aufgaben für den Fahrer führen. Diese Veränderung geht mit der steigenden $\mathrm{Au}-$ tomatisierung einher, da immer mehr Aufgaben von dem Fahrzeug in vielen Situationen übernommen werden können. Die neue beziehungsweise veränderte psychologische Situation wird sich auch in veränderten physiologischen Eigenschaften beim Menschen gemäß der Psychophysiologie widerspiegeln. Nachfolgend wird die psychologische
Situation näher beschrieben und gezeigt, welche messbaren physiologischen Effekte damit in Zusammenhang gebracht werden können.

\subsection{Vigilanz}

Um den Begriff der Vigilanz besser im Kontext des automatisierten Fahrens einordnen zu können, sollte zunächst ein allgemeinerer Blick auf das psychologische Verhalten beim Fahren geworfen werden. Bei dem manuellen Fahren handelt es sich um eine Daueraufmerksamkeitsaufgabe. Der Fahrer muss ständig die Umgebung erfassen und sowohl die Quer- als auch die Längsbewegung des Fahrzeugs regeln. Da das Fahrzeug in 0,7 s die Fahrspur auf gerader Strecke zur Seite soweit verlassen kann, dass ein Fahrer eingreifen würde, ist gerade bei der Querführung des Fahrzeugs eine hohe Reizrate vorhanden [11]. Der Fahrer reagiert in kurzen Abständen auf den Reiz, dass das Fahrzeug die Fahrspurmitte verlässt. Ebenso wird in etwas längeren Zeitintervallen die Geschwindigkeit des Fahrzeugs kontrolliert und angepasst.

Bei einer Automatisierung ab SAE Level 2 entfallen beide Aufgaben zur Fahrzeugführung. So kann es dazu kommen, dass die Reizrate für den Menschen wesentlich geringer ausfällt, da nur noch selten auf ein Ereignis reagiert werden muss. Die Vigilanz beschreibt genau diese Situation: Die Fähigkeit, auch nach einer längeren Zeit noch auf schwache und seltene Reize reagieren zu können. Schon in [17] wird gezeigt, dass dieser Effekt, also nachlassende Reaktionsfähigkeit auf Reize, bei Signalentdeckungsaufgaben mit geringen Reizen auftreten kann. Die Aufgabe besteht in der zitierten Untersuchung darin, einem Lichtpunkt zu folgen. Wenn dieser Lichtpunkt eine Position überspringt, soll die Versuchperson dies mit einem Tastendruck angeben. So ist diese Aufgabe nicht auf die Anwendung des Fahrens bezogen. Die Reizstärke ist in dem Fall des Fahrzeugführens schwieriger zu beurteilen als die Reizfrequenz, da es noch keine Untersuchungen in diesem Kontext gibt. Wie die Stärke eines Reizes gemessen wird und wie gut ein Fahrer auf diesen auch nach längerer Zeit regieren kann, ist Gegenstand der Forschung.

In der Literatur, wie zum Beispiel von [21], wird der Begriff Vigilanz manchmal als Synonym für den Begriff der Müdigkeit verwendet. Hier sollte jedoch, wie von [7] zusammengefasst, zwischen der Vigilanz und Müdigkeit unterschieden werden, da die Müdigkeit eher als ein Auslöser für den Verlust von Vigilanz (Hypovigilanz) beschrieben werden kann. Vigilanz grenzt sich dahingehend von der Müdigkeit durch die genaue psychologische Definition $a b$, bei der eine verminderte Reaktion auf schwache seltene Reize angegeben wird. Dies kann auch auftreten, wenn es wenige Anzeichen für Müdigkeit, durch zum Beispiel Schlafmangel, gibt. 


\subsection{Physiologische Eigenschaften}

Die Psychophysiologie beschreibt, wie sensorische, motorische, kognitive Fähigkeiten und Emotionen im Zusammenhang mit der Hirntätigkeit, dem Kreislauf, der Atmung und Hormonausschüttung stehen. Dabei existieren jedoch starke individuelle Unterschiede, sodass oftmals der Zusammenhang lediglich qualitativ bestimmt werden kann. So gibt es zu verschiedenen Körperfunktionen bereits Thesen für den Zusammenhang zum psychologischen Zustand der Vigilanz.

\subsubsection{Gehirn}

Von der Gehirnaktivität ausgehend lassen sich Aktivitäten in der Hirnrinde (Kortex) durch an der Schädeloberfläche auftretende Spannungsunterschiede (Gehirnwellen) bestimmen (EEG - Elektroenzephalografie). Diese Signale werden oft für eine objektive Messung des psychologischen Zustands eines Menschen herangezogen, wie es [20] für verschiedene Anwendungen zeigt. Hierbei wird das Frequenzspektrum der Spannungswerte in fünf überlappende Frequenzbänder [10] aufgeteilt:

Delta $(\delta)[1-4 \mathrm{~Hz}]$, Theta $(\theta)[4-8 \mathrm{~Hz}]$, Alpha $(\alpha)[7,5-$ $13 \mathrm{~Hz}]$, Beta $(\beta)[13-30 \mathrm{~Hz}]$ und Gamma $(\gamma)[30-44 \mathrm{~Hz}]$. Die einzelnen Frequenzen lassen sich, wie von [34] gezeigt, mit verschiedenen Charakteristika in Zusammenhang bringen. So wird bei dem hier vorliegenden Versuch erwartet, dass eine höhere Theta Aktivität zu finden ist, weil diese in Phasen von Vigilanzminderung auftreten kann. Ebenso ist es möglich, dass eine vorübergehend erhöhte Beta Aktivität festzustellen ist, weil diese mit psychischer Belastung und Vigilanzabnahme (subviglile Beta-Aktivität) in Zusammenhang gebracht werden kann. Eine erhöhte Gamma Aktivität wird dabei mit einem Wachzustand in Zusammenhang gebracht.

\subsubsection{Herz}

Das Herz kann bei verschiedenen psychologischen Situationen den Rhythmus verändern und anpassen. Dabei sind zwei Maße ausschlaggebend für die Analyse des Herzrhythmus, die Herzfrequenz $F$ und die Herzraten-Variabilität $H$. Bei der Herzfrequenz $F$ geht es grundlegend um die Häufigkeit von Herzschlägen pro Zeitspanne. In den meisten Fällen bezieht sich dieses Maß auf Herzschläge pro Minute mit der Einheit beats per minute (bpm). Die HerzratenVariabilität wird, wie in [25] zusammengefasst, zum Beispiel durch eine Analyse der Zeitdifferenzen zwischen einzelnen Herzschlägen charakterisiert. Dabei berechnen sich die Zeiten zwischen einzelnen Herzschlägen $r_{i}$ über eine Auswertung der Kurve des Elektrokardiogramm, indem die Zeit zwischen den so genannten R-Wellen in dem Signal bestimmt wird [6]. So kann der Wert der Herzraten-Variabilität $H$ über eine Zeitspanne $T$ mit den Zeiten zwischen den Herzschlägen $r_{i}$ wie folgt berechnet werden:

$H=\sqrt{\sum_{i=0}^{T}\left(r_{i+1}-r_{i}\right)^{2}}$

In dieser Arbeit wird für die Zeitspanne $T=60 \mathrm{~s}$ verwendet.

In [12] wird gezeigt, dass bei zunehmender mentalen Belastung die Herzraten-Variabilität abnimmt. So wird bei diesem Merkmal erwartet, dass der Wert $H$ in der vorliegenden Arbeit im Verlauf des vorgestellten Versuchs zunehmen sollte, da die mentale Belastung als sehr niedrig eingeschätzt wird.

\subsubsection{Augen}

Die Augen spiegeln durch verschiedene Signale eine Änderung des psychologischen Zustands wider. Sowohl die Augenbewegung und Fixation als auch die Pupillenweite und Schließdauer [18] sind hilfreich für die Bestimmung eines Zusammenhangs zwischen einer physiologischen Veränderung und der kognitiven Belastung des Fahrers. In der vorliegenden Arbeit liegt der Fokus auf der Augenschließdauer, da sie leicht aus einiger Entfernung berührungslos durch eine Kamera bestimmbar ist. Üblicherweise wird, wie in [29] gezeigt, die prozentuale Zeit (englisch: PERcentage of Eyelid CLOSsure - PERCLOS) in der das Auge zu mindestens $80 \%$ geschlossen ist, als Maß herangezogen. Dabei wird als Zeitintervall $60 \mathrm{~s}$ vorgeschlagen und zudem eine Korrelation zwischen dem Ansteigen des Wertes und der Müdigkeit festgestellt. Dieser Zusammenhang wird in [26] konkret bei dem Führen eines Fahrzeugs untersucht und mit anderen Signalen, unter anderem den Gehirnwellen, kombiniert. Dabei kann festgestellt werden, dass die Augenschließdauer (PERCLOS-Wert) mit der Müdigkeit beim Fahren eines Fahrzeugs korreliert. Dazu wird die Müdigkeit, neben dem PERCLOS-Wert, über eine subjektive Einschätzung der Versuchspersonen durch die Karolinska Sleepiness Scale (KSS) [1] bestimmt, sodass eine Korrelation zwischen den verschiedenen Messmethoden gezeigt werden kann. Die Korrelation ist beim PERCLOSE-Wert jedoch schwächer im Vergleich zu den Gehirnwellen ausgeprägt.

\section{Vigilanz-Studie}

Die für die Untersuchung der Vigilanz zugrunde liegende Studie bestand sowohl aus Befragungen der Versuchspersonen als auch einem praktischen Teil mit einer Aufgabe in einem statischen Fahrsimulator. Im Folgenden wird der Versuchsablauf und Hintergrund genauer erläutert und zum 
Schluss des Abschnitts werden die Ergebnisse der Befragung zusammengetragen und ausgewertet.

\subsection{Studiendesign}

Bei dem praktischen Teil der Vigilanz-Studie handelte es sich um einen Versuch in einem statischen Fahrsimulator. Bei diesem wurde eine längere automatisierte Autobahnfahrt simuliert und zusätzlich war eine Signalentdeckungsaufgabe zu bewältigen. Es ging um die kontinuierliche Messung der Aufmerksamkeit während des Versuchs, ohne eine Übergabe zwischen dem automatisierten und manuellen Fahren, da dies einen starken Reiz mit sich bringen würde. Der Aufbau bestand aus einem Bildschirm frontal vor der Versuchsperson und einer Sitzkiste, in der zwei Autositze sowie Lenkrad und Pedalerie angebracht sind. Zudem bietet der Simulator die Möglichkeit verschiedene Sensoren flexibel zu platzieren. In der Fahrsimulation fuhr das Fahrzeug auf einer über $100 \mathrm{~km}$ langen Autobahnstrecke mit maximal $130 \frac{\mathrm{km}}{\mathrm{h}}$ auf zwei- oder dreispurigen Streckenabschnitten [5]. Zwischen jedem Streckenabschnitt von maximal $10 \mathrm{~km}$ wurde eine Abfahrt oder ein Autobahnkreuz passiert. Es wurde eine Verkehrssituation mit mittlerem Verkehrsaufkommen auf beiden Fahrbahnseiten simuliert, welche bei jeder Simulation zufällig neu im Umkreis des Fahrzeugs generiert wurde. Um die Verkehrsbedingungen für jede Versuchsperson gleich zu halten, wurde für diesen Versuch ein Video verwendet.

Dazu wurden synthetische Straßenschilder am Fahrbahnrand in die Fahrsituation integriert, um eine Vigilanz-Aufgabe, wie in [17] beschrieben, einbringen zu können. Die Schilder waren generell etwa $900 \mathrm{~m}$ voneinander entfernt mit einer Varianz von $90 \mathrm{~m}$ um einem Gewöhnungseffekt entgegenzuwirken. Die Schilder wurden aus zwei Gruppen ausgewählt: Schilder, die gewöhnlich auf der Autobahn zu finden sind und Schilder, die nicht in einer realen Fahrsituation auf der Autobahn zu sehen sind. Auf die zweite Gruppe von Schildern sollte die Versuchsperson mit einem Tastendruck reagieren. Um die Aufgabe am Anfang leicht und später schwieriger zu gestalten, wurden in der ersten Hälfte der Versuchszeit $40 \%$ und danach nur noch $20 \%$ der Schilder aus der zweiten Gruppe gewählt. Das bedeutet, dass etwa alle $25 \mathrm{~s}$ ein Schild am Straßenrand zu sehen war, aber am Ende nur alle zwei bis drei Minuten auf ein Schild reagiert werden musste. Dabei wird die These aufgestellt, dass sich die Reaktionszeiten zum Ende des Fahrversuchs erhöhen. Diese ist also analog zu der These aus [17] gestaltet, nur auf eine Situation im Straßenverkehr bezogen. Eine Erfüllung dieser These würde die Definition des Begriffes Vigilanz entsprechen.

Um den Versuchsablauf besser nachvollziehen zu können, folgen nun Erläuterungen zu den einzelnen Schritten des Versuchs: Zuerst wurde ein Fragebogen zur Person

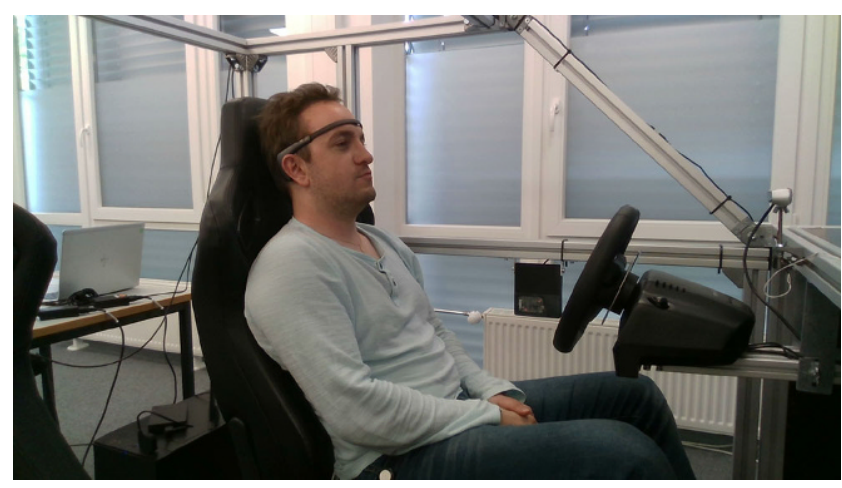

Abb. 1 Versuchsperson in dem Versuchsaufbau

ausgefüllt, bei dem nach Alter, Geschlecht, Fahrerfahrung und aufmerksamkeitsbeeinflussenden Eigenschaften (Schlaf, Kaffeekonsum, usw.) gefragt wurde. Dabei wurde für das Befinden und die Schlafqualität der letzten Nacht eine fünfstufige Skala wie folgt ausgelegt: Sehr gut, gut, neutral, schlecht und sehr schlecht. Danach wurden die kontaktbehafteten Sensoren (EEG-Stirnband und HerzBrustgurt) am Probanden platziert und geprüft, indem die Rohsignale und teilweise deren Varianz angezeigt wurden. Als nächstes wurden die Versuchsinstruktionen zusammen mit der Signalentdeckungsaufgabe erklärt. Nachdem die Versuchsperson, wie in Abb. 1 gezeigt, in dem Fahrsimulator den Platz eingenommen hatte, wurde die Messung aller Sensoren gestartet. Dazu startete das Video automatisch, sodass die automatisierte Versuchsfahrt begann, wobei die Versuchsperson nur die Signalentdeckungsaufgabe zu erfüllen hatte. Nach der Versuchsfahrt verließ die Versuchsperson den statischen Fahrsimulator, legte die Sensoren wieder ab und beantwortete noch einen Fragebogen zur subjektiven Bewertung der Fahrt bezogen auf die erlebte Langeweile, Konzentration-, Anstrengungs- und Müdigkeitswahrnehmung. Bei diesem Fragebogen wurde eine fünfstufige Skala wie folgt gewählt: Sehr, Ziemlich, Mittelmäßig, Etwas und Gar nicht.

\subsection{Studienergebnisse}

An der Studie haben neun Versuchspersonen mit einem Alter von $\varnothing 27,7$ Jahren mit einer Standardabweichung (STD) von 3,6, die seit $\varnothing 9,0$ Jahren (STD 4,2) ihren Führerschein besitzen, teilgenommen. Keiner dieser Personen war an dem Versuchsdesign beteiligt. Der Versuch hat vorwiegend nachmittags stattgefunden und alle Versuchspersonen besaßen mindestens die Führerscheinklasse B. In der Gruppe der Probanden waren 6 Personen mit einer Sehhilfe vertreten, von denen eine Person Kontaktlinsen und der Rest eine Brille trug. Die Schlafzeit in der Nacht vor dem Versuch lag bei $\varnothing 6,89$ Stunden (STD 0,42 Stunden). Die Ergebnisse zu der Schlafqualität und dem aktuellen Befinden sind in Abb. 2 


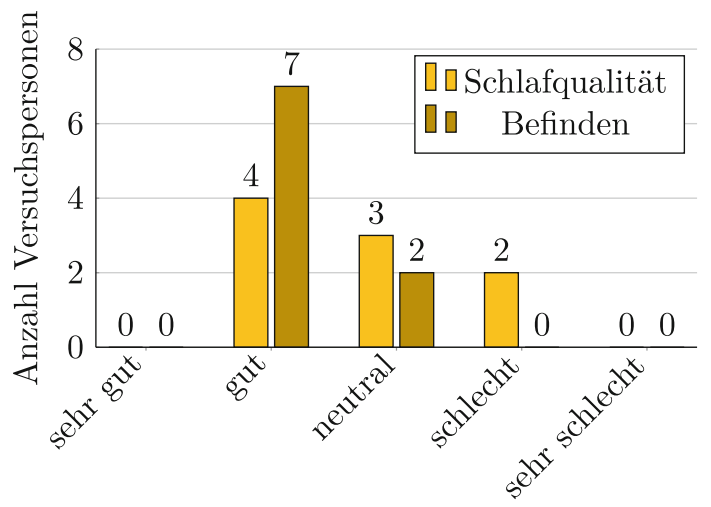

Abb. 2 Auswertung der Befragung zu Schlafqualität und Befinden vor dem Versuch

dokumentiert. Dabei wird deutlich, dass die Schlafqualität vor dem Versuch bei etwa 44\% der Versuchspersonen als gut empfunden wurde und $22 \%$ der Versuchspersonen den Schlaf als schlecht bewerteten. Das Befinden vor Antritt der Fahrt ist bei $78 \%$ der Versuchspersonen als gut bewertet worden und der Rest der Gruppe bewertete das Befinden als neutral.

Die Befragung nach der Fahrt wird in Abb. 3 aufgezeigt. Hier wird deutlich, dass bei allen Versuchspersonen Langeweile aufgetreten war. Ein Drittel bewertete das Auftreten mit Sehr stark, ein Drittel mit Ziemlich stark und ein Drittel mit Mittelmäßig stark. Die Konzentrationseinschätzung ist fast symmetrisch um die Angabe Mittelmäßig verteilt, bis auf eine Versuchsperson, die sich Sehr stark konzentrieren musste, um die Signalentdeckungsaufgabe zu bewältigen. Die Anstrengungswahrnehmung war von drei Versuchspersonen mit Mittelmäßig eingestuft worden, drei Versuchspersonen schätzten diese auf Ziemlich, eine Person auf Sehr und zwei Versuchspersonen spürten die Anstrengung Gar nicht während des Versuchs. Die Müdigkeit trat bei allen Versuchspersonen in der subjektiven Bewertung auf. Dabei schätzten sich selber $78 \%$ der Gruppe mit Ziemlich müde, $11 \%$ mit Mittelmäßig müde und weitere $11 \%$ mit Sehr müde ein.

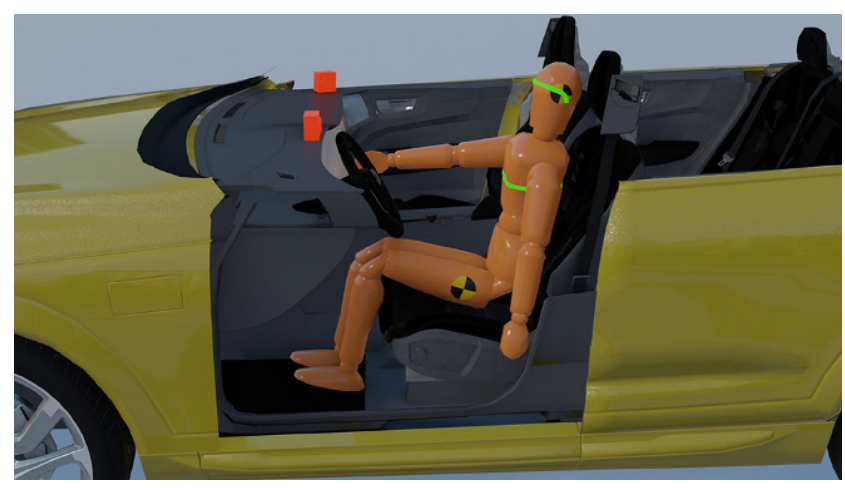

Abb. 4 Sensoraufbau

\section{Sensoraufbau}

Um die in Abschn. 2.2 beschriebenen Körperfunktionen erfassen zu können, wurde ein Sensorsystem benötigt. Ein Überblick über mögliche Sensoren in einem Fahrzeug wird in [15] gegeben. Wobei in dem vorliegenden Beitrag der Fokus auf kontaktlose Sensoren gelegt wurde. Bei [23] werden verschiedene Messmethoden für die Müdigkeit zusammengetragen, sodass sich auch in dem vorliegenden Beitrag der Sensoraufbau an diesen Beispielen orientierte.

Bei der hier vorgestellten Studie wurde eine Kombination aus verschiedenen Sensoren verwendet. Dabei standen sowohl kontaktlose als auch kontaktbehaftete Sensoren zur Messung der physiologischen Merkmale aus Abschn. 2.2 zur Verfügung. In Abb. 4 sind die Positionen der Sensoren angegeben. Es wurden zwei Kameras auf dem Armaturenbrett, ein Sensor am Brustkorb und ein Sensor an der Stirn der Versuchsperson in dem Sensoraufbau verwendet. In der Abb. 4 sind die kontaktlosen Kameras in Rot und die kontaktbehafteten Sensoren in Grün farblich hervorgehoben. Dazu erfolgte die Bestätigung der Signalentdeckungsaufgabe durch Knopfdruck an dem angebrachten Lenkrad.
Abb. 3 Auswertung der Befragung zu psychischen Eigenschaften nach dem Versuch

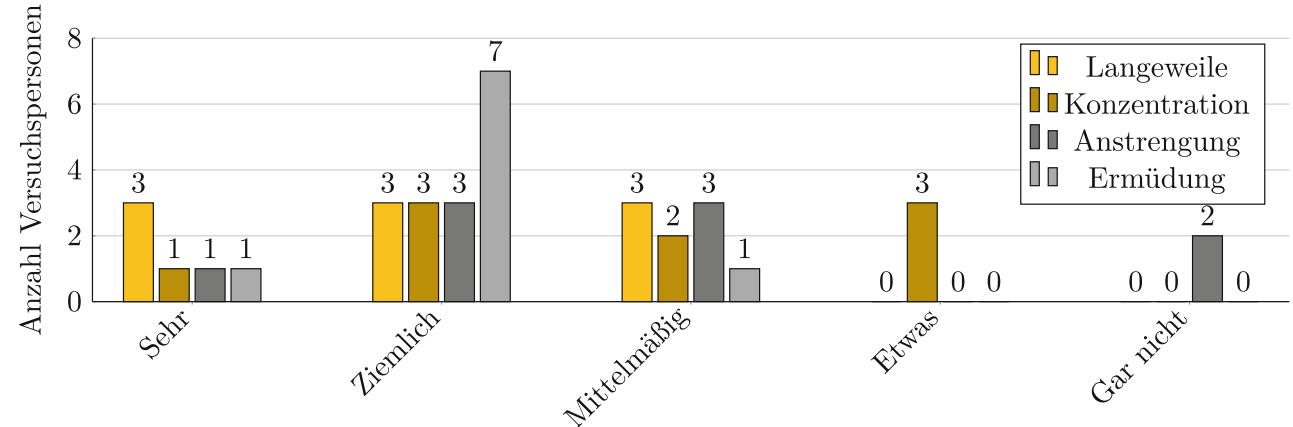




\subsection{Reaktionszeiten}

Zur Messung der Reaktionszeiten wurde der Knopfdruck an dem Lenkrad verwendet. Um zu bestimmen worauf die Versuchsperson reagiert, wurde die Startzeit des Videos, welches den Versuchspersonen eine automatisierte Fahrt vermitteln sollte, ermittelt. Mit dieser konnten einzelne Bilder des Videos mit den Zeitpunkten eines Knopfdrucks in Zusammenhang gebracht werden. Um nun die Reaktionszeiten zu bestimmen wurde das Video in Einzelbilder aufgeteilt. Daraufhin wurde das Bild aus den Einzelbilder bestimmt, auf dem das Straßenschild klar von anderen Straßenschildern unterschieden werden konnte, welches dann als Referenzbild und Zeitpunkt für die Zeitmessung diente. Zuletzt wurde die Differenz zwischen dem Zeitpunkt des Knopfdrucks und dem Zeitpunkt vom Erscheinen des Referenzbilds gebildet.

\subsection{Elektroenzephalogramm}

Für die Messung der Gehirnwellen wurde ein Elektroenzephalogramm (EEG) benutzt. In [8] wird beschrieben, dass diese Messung die besten Ergebnisse für eine Fahrerzustandseinschätzung gibt. Dabei wird darauf hingewiesen, dass der Sensor kontaktbehaftet ist und zusätzlich oftmals ein Kontaktgel verwendet wird, sodass die Versuchsperson abgelenkt werden könnte. Um bei der vorliegenden Studie die Messung möglichst nichtinvasiv zu halten, wurde ein kabelloses Stirnband mit trockenen Elektroden benutzt. Das verwendete Stirnband ist mit vier Elektroden und einer Referenzelektrode ausgestattet. Dies ist ausreichend, da keine qualitative Aussage über die örtliche Herkunft von Gehirnwellen gegeben werden soll. Wenn eine starke Änderung der Gehirnwellen vorliegt, dann kann von verschiedenen Zuständen des Gehirns ausgegangen werden, wie [33] schon für wenige Elektroden zur Müdigkeitserkennung von Zugfahrern zeigt und verifiziert. Zudem bietet der verwendete Sensor noch weitere Messwerte wie Gyroskop-, Beschleunigungswerte und eine Photoplethysmographie (Herzschlag). Weil in der vorliegenden Arbeit die Änderung über sehr große Zeiträume (> $30 \mathrm{~min}$ ) von Interesse sind, wurde eine Mittlung über einen Zeitraum von 10 s vorgenommen. Damit wurden besonders Messausreißer und Störungen durch Blinzeln und Kopfbewegungen gemildert, die sich meistens in einem Zeitraum von unter 10 s abspielen. Dabei wurde die Zeitspanne von 10 s empirisch bestimmt.

\subsection{Elektrokardiogramm}

Da ein Elektrokardiogramm über elektrische Signale vom Herzen gemessen wird, musste dieses mit einem kontaktbehafteten Sensor durchgeführt werden. Hier wurde ebenso, wie bei dem EEG, ein kabelloser Sensor verwendet, um keine Störungen durch Verkabelungen zu erhalten. Dieser Sensor misst sowohl die Herzschläge pro Minute, als auch die Zeiten zwischen den einzelnen Herzschlägen (R-R-Intervalle). Die Messwerte wurden mit $1 \mathrm{~Hz}$ ausgegeben, wobei die Zeit zwischen einzelnen Herzschlägen gegebenenfalls unter eine Sekunde betragen kann, sodass mehrere Messwerte gleichzeitig ausgegeben werden konnten.

\subsection{Kamera}

Um eine kontaktlose Beobachtung des Fahrers zu erhalten, wurden, wie bereits aufgezeigt, zwei Kameras verwendet. Eine war, wie in Abb. 4 zu sehen, hinter dem Lenkrad und die zweite an der A-Säule auf der Beifahrerseite auf dem Armaturenbrett befestigt. Die Kameras nahmen Farbbilder mit einer Auflösung von 1920 mal 1080 Pixel mit 30 Bildern pro Sekunde auf. Um die Informationen über das Auge zu extrahieren, wurde im ersten Schritt der in [32] beschriebene Algorithmus verwendet. Dieser liefert Schlüsselpunkte der Augen in Pixelkoordinaten, mit denen dann die Breite des linken Auges $b_{1}$ und des rechten Auges $b_{\mathrm{r}}$, sowie die Höhe des linken Auges $h_{1}$ und des rechten Auges $h_{\mathrm{r}}$, jeweils als Pixelabstand, bestimmt werden können. Aus diesen Werten wird dann wie folgt das Höhen- zu Breitenverhältnis $r$ eines Datenpunkts bestimmt:

$r=\frac{\frac{h_{1}}{b_{1}}+\frac{h_{\mathrm{r}}}{b_{\mathrm{r}}}}{2}$

Beispielhaft ist in Abb. 5 das Höhen- zu Breitenverhältnis $r$ einer Versuchsperson abgebildet, wobei in diesem Fall der Wert bei geöffnetem Auge etwa 0,3 betrug und bei geschlossenem Auge bei etwa 0,07 lag. Um Messausreißer aus dem Signal $r$ zu entfernen, wurden alle Messwerte, die mehr als 0,05 nach oben, da nur Augenschließungen in diesem Fall interessant sind, von dem Mittelwert (in dem gezeigten Fall $\varnothing r=0,27)$ abweichen auf den Mittelwert

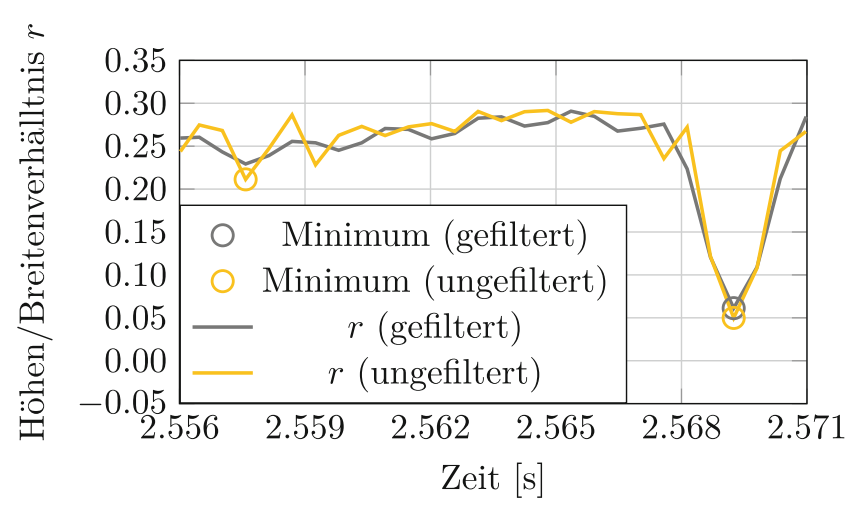

Abb. 5 Bestimmung der Augenschließung bei gefiltertem und ungefiltertem Signal 


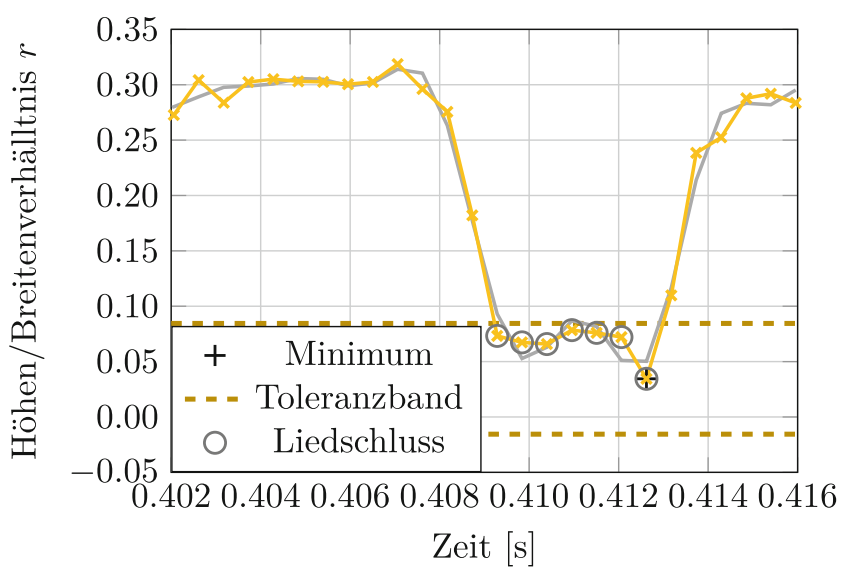

Abb. 6 Höhen- zu Breitenverhältnis für eine Augenschließung und Bestimmung der Schließzeit

gesetzt. Dieses Signal war jedoch immer noch verrauscht, sodass ein Tiefpassfilter mit einer Grenzfrequenz von $15 \mathrm{~Hz}$ (halbe Abtastfrequenz) angewendet wurde, welches durch die graue Linie in der Abb. 5 visualisiert wird. Dieses Filter dient dazu besser zeitliche Minima zu finden, um eine Augenschließung von Rauschen zu unterscheiden, wie bei 2,557 s sichtbar wird. Denn hier sorgte das Rauschen für eine Fehlerkennung, welche bei dem gefilterten Signal nicht auftrat. Ebenso wäre ein gleitender Mittelwert zur Glättung des Signals geeignet, wobei auch hier ein Parameter mit der Fensterlänge des Filters einzustellen wäre. Dazu wurden nur Minima mit einer Schartenhöhe von mindestens 0,07 als Augenschließung gezählt, sodass keine Nebenminima durch das Rauschen hinzukamen.

Um die Schließdauer zu bestimmen, wurden von dem Minimum nach links und rechts alle Messpunkte markiert, die in einem Toleranzband von 0,05, wie in der Abb. 6 gezeigt, um den Wert des Minimums lagen. Zuletzt wurde noch die Anzahl dieser markierten Messpunkte durch die Anzahl der Messungen in dem Intervall von $60 \mathrm{~s}$ (wie in Abschn. 2.2.3 beschrieben) bestimmt, um die prozentuale Schließdauer, also den PERCLOS-Wert, zu erhalten.

Dieses Verfahren wurde mit der manuellen Auswertung von Bildern aus dem hier generierten Datensatz von drei unterschiedlichen Versuchspersonen verifiziert. Hierzu wurden jeweils über eine Minute die Einzelbilder gezählt, bei denen das Auge zu mehr als $80 \%$ geschlossen war, und durch die Gesamtzahl der Bilder in der Minute geteilt. Dabei stellt sich eine Abweichung von durchschnittlich 0,812 Prozentpunkten zu dem im vorliegenden Beitrag vorgestellten Verfahren heraus. Hierbei lag das in dem vorliegenden Beitrag vorgestellte Verfahren vom Wert jedoch immer unter der manuellen Auswertung.

\section{Datenauswertung}

Da die Größe der Stichprobe in der vorliegenden Studie sehr gering ist, wird an dieser Stelle das methodische Vorgehen zur Datenverarbeitung analysiert. So wird trotz der geringen Größe des Datensatzes ein künstliches neuronales Netzwerk vorgestellt und trainiert, um eine mögliche Datenverarbeitung für einen größeren Datensatz vorzubereiten. Die Datenauswertung wird offline umgesetzt und dient zur Analyse, ob und mit welchen Sensoren eine Erkennung des psychologischen Zustands möglich ist. Zunächst wird in diesem Abschnitt beschrieben, wie die Daten der verschiedenen Sensoren zusammengeführt werden, um sie dann mit einem künstlichen neuronalen Netz zu klassifizieren. Hierbei soll im Fokus stehen, welches Klassifikationsergebnis des psychologischen Zustands bei verschiedenen Kombinationen von Sensoren, also verschiedenen Merkmalen, auftritt. Dazu werden beim Training Daten einzelner Sensoren aus der Menge der Trainingsdaten genommen und die Ergebnisse auf ungesehenen Testdaten miteinander verglichen.

\subsection{Datenvorbereitung}

Zunächst werden aus den einzelnen Rohsignalen die in Abschn. 2.2 beschriebenen Merkmale berechnet. Da die Sensoren unterschiedliche Abtastraten aufweisen, muss der Datensatz auf eine gemeinsame Zeitskala mit definierten Abständen gebracht werden. Dabei wird eine Abtastzeit von $10 \mathrm{~s}$ gewählt, da die Mittlung der Gehirnwellen die kleinste Abtastzeit aller Messgrößen darstellt. Die Anderen Messgrößen werden in einem Intervall von $60 \mathrm{~s}$ bestimmt. Um die Messwerte für diese festen Zeitschritte zu bestimmen, wird eine lineare Interpolation der Messdaten zwischen benachbarten Zeitstempeln durchgeführt. Das bedeutet, dass die Messung einer Versuchsperson über 45 Minuten (2700s) 270 Datenpunkte erzeugt. Da in der ersten Minute noch starke Bewegungsartefakte das EEG stören, werden diese Messwerte gelöscht, sodass 264 Datenpunkte weiter verarbeitet werden können. Für das später verwendete überwachte Lernverfahren ist es notwendig, die Daten in die verschiedenen Klassen, hier Vigilant und Hypovigilant genannt, einzuordnen. Die Einordnung sollte über die Reaktionszeiten der Versuchspersonen erfolgen. In Abb. 7 sind die durchschnittlichen Reaktionszeiten mit den zugehörigen Standardabweichungen dargestellt. Zu erkennen ist, dass mit fortdauerndem Versuch der Trend eher zu kleineren Reaktionszeiten geht. Eine negative Reaktionszeit ist dabei möglich, da der Zeitpunkt des visuellen Reizes auf Grundlage von Einzelbildern bestimmt wird, auf denen das Straßenschild klar von anderen unterschieden werden kann und die Versuchspersonen vor diesem Zeitpunkt den Knopf drücken können. Die Versuchspersonen scheinen das Er- 


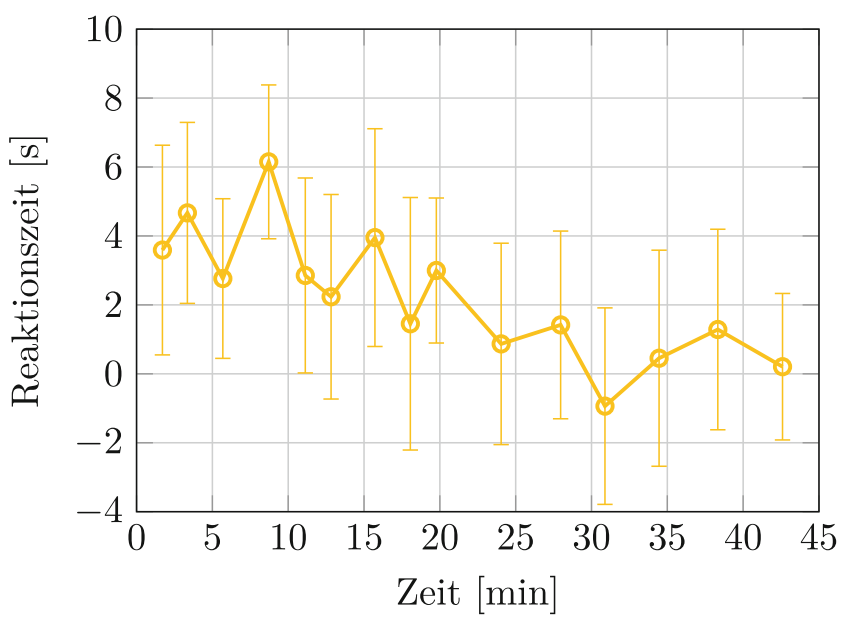

Abb. 7 Reaktionszeiten der Versuchspersonen auf die Straßenschilder

kennen der Schilder durch Merkmale wie Farbe oder Größe zu lernen und sind so in der Lage, die Schilder schneller voneinander zu unterscheiden.

Die Reaktionszeiten sollten direkt als psychologischer Versuch den exakten Nachweis zur psychologischen Definition von Vigilanz erbringen. Da sich dies aber nicht durch die Ergebnisse der Reaktionszeiten abzeichnete, wurde die vorgesehene Datenverarbeitung dennoch bei einer Veränderung in den kognitiven Eigenschaften getestet. Hier ist die Situation, durch Länge und Art, vermutlich dennoch mit einer Vigilanzaufgabe vergleichbar. Nachfolgend wird bei einer kognitiven Veränderung zwischen den hier so genannten Klassen Vigilant und Hypovigilant unterschieden, ohne einen psychologischen Nachweis für diese zwei Zustände zu haben.

Dabei wird bei einer großen Veränderung der $\beta$ - und $\gamma$ Wellen auf einen Zustandswechsel geschlossen.

So kann in Abb. 8 das Vorgehen an einem Beispiel aus den Trainingsdaten gesehen werden, bei dem die Gehirn-

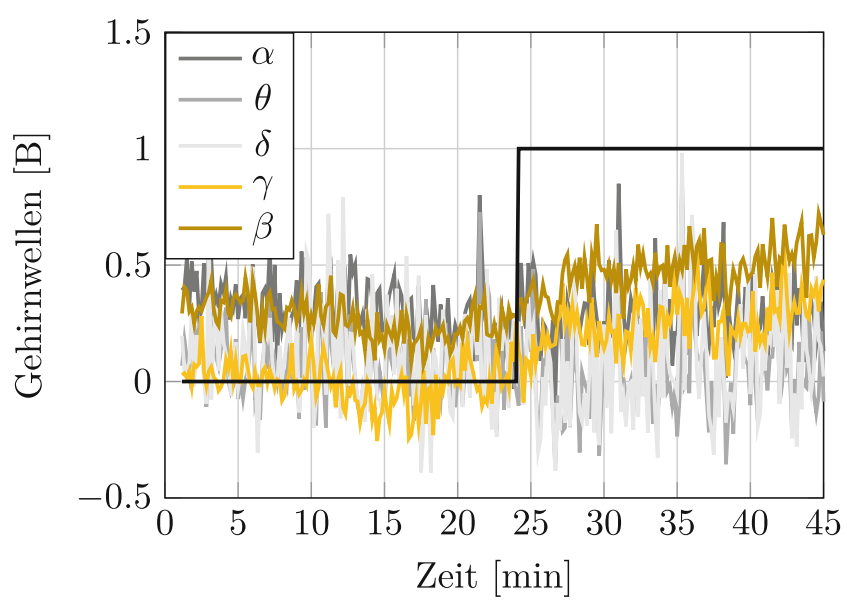

Abb. 8 Gehirnwellen einer Versuchsperson mit eingezeichneter Beschriftung (Ground Truth) wellen ab etwa Minute 24 eine größere Veränderung aufweisen, da die $\beta$ - und $\gamma$-Wellen im Vergleich zu den anderen Gehirnwellen ansteigen. Dabei wird darauf geachtet, dass die Mittelwerte sich deutlich von einander unterscheiden (Mittelwertänderung größer, als Varianz im Signal). So wird in diesem Fall auch die Kennzeichnung des kognitiven Fahrerzustands, in schwarz dargestellt. In diesem Zeitpunkt wird die Beschriftung von dem hier so genannten Zustand Vigilant zu Hypovigilant geändert, welche aber nicht nachgewiesen werden konnten. Diese Annahme fußt lediglich auf die oben erwähnte subvigile $\beta$-Aktivität, welche mit einer Verminderung der Vigilanz nach [34] einhergeht. Da$\mathrm{zu}$ ist in [7] zusammengefasst, dass eine $\gamma$-Aktivität mit einem Wachzustand verbunden werden kann, sodass hier möglicherweise keine Müdigkeit, sondern eine angestrengte Aufmerksamkeit mit Wachheit verbunden sein könnte. Leider gibt es aber zu diesem Effekt keine weiteren Untersuchungen in der Literatur, sodass hier ein recht explorativer Ansatz gewählt wird, der im Nachhinein noch von weiteren Studien belegt werden sollte. Die weiteren Daten werden nach dem gleichen Prinzip manuell beschriftet, was aufgrund der individuellen Unterschiede nicht immer präzise durchgeführt werden kann, wie es für $\beta$-Aktivitäten schon von [34] beschrieben wurde. So kann bei einigen Probanden der Zeitpunkt des Zustandswechsels zeitlich nur grob bestimmt werden, da die Änderung der Gehirnwellen bei jeder Versuchsperson unterschiedlich stark ausgeprägt sind. Es gibt durch die starken Unterschiede zwischen den einzelnen Versuchspersonen keine festen Schwellenwerte, jedoch sind qualitative Aussagen der Versuchspersonen nach dem Versuch mit in die Bewertung eingeflossen. Eine andere Möglichkeit Unterschiede auszumachen, wäre die Analyse einer signifikanten Änderung in den Sensordaten, jedoch sind hierfür nicht genügend Datenpunkte pro Proband vorhanden.

Nach dem Erkennen von starken Änderungen und Beschriften der Daten muss noch dafür gesorgt werden, dass die Werte jedes Sensors von dem Netzwerk gleich verarbeitet werden können. Dazu werden alle Daten von dem Mittelwert befreit und über die Varianz normiert. So wird sichergestellt, dass kein Merkmal vom Netzwerk bevorzugt wird und die Gewichte sich in der gleichen Größenordnung befinden. Die Daten der neun Versuchspersonen werden in Trainings- und Testdaten aufgeteilt. Dabei werden die Daten von acht Versuchspersonen für die Trainingsdaten verwendet und die Daten einer Versuchsperson als Testdaten herausgestellt. In den folgenden Abschnitten bleibt diese Aufteilung gleich.

\subsection{Struktur des künstlichen neuronalen Netzwerks}

Um die Klassifizierung gleichzeitig mit einer Fusion der Merkmale auf hoher Abstraktionsebene zu realisieren, wer- 


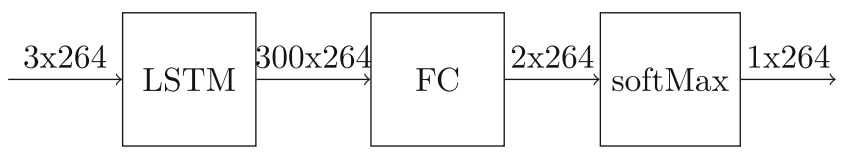

Abb. 9 Struktur des künstlichen neuronalen Netzwerks

Tab. 1 Merkmale für das Training der Datenverarbeitung

\begin{tabular}{lll}
\hline & Körperteil & Merkmal \\
\hline 1 & Herz & Herzfrequenz $F$ \\
2 & & Herzraten-Variabilität $H$ \\
\hline 3 & Augen & PERCLOS \\
\hline
\end{tabular}

den in dieser Arbeit künstliche neuronale Netzwerke verwendet. Da davon ausgegangen werden kann, dass sich Müdigkeit kumuliert, ist es wichtig, den zeitlichen Verlauf der Merkmale zu berücksichtigen, sodass bei der Struktur die Wahl auf Long Short-Term Memory (LSTM) Netzwerke [9] gefallen ist. Diese wurden zum Beispiel auch schon von [31] zur Erkennung von Müdigkeit so ähnlich wie in dem vorliegendem Beitrag verwendet. In Abb. 9 ist die Struktur des vollständigen Netzwerks abgebildet, die aus einer LSTM-Schicht, einer vollständig verknüpften Schicht (FC) und einer SoftMax-Schicht besteht. Der Eingang wird mit Sequenzen mit einer Länge von 264 Messpunkten, wie in Abschn. 5.1 beschrieben, belegt. Die Anzahl der Sequenzen bestimmt sich aus der Anzahl der Merkmale, die für die Klassifizierung benutzt werden. In diesem Fall ist das Netzwerk mit den drei Merkmalen aus der Tab. 1 dargestellt, die sich zu zwei Merkmalen aus dem Herzen und einem Merkmal aus den Augen aufteilen. Die LSTM-Schicht besteht aus 300 verstecken Einheiten, die parallel mit den Sequenzen gespeist werden. Heraus kommen 300 Sequenzen, die durch die LSTM-Schicht verarbeitet wurden, die

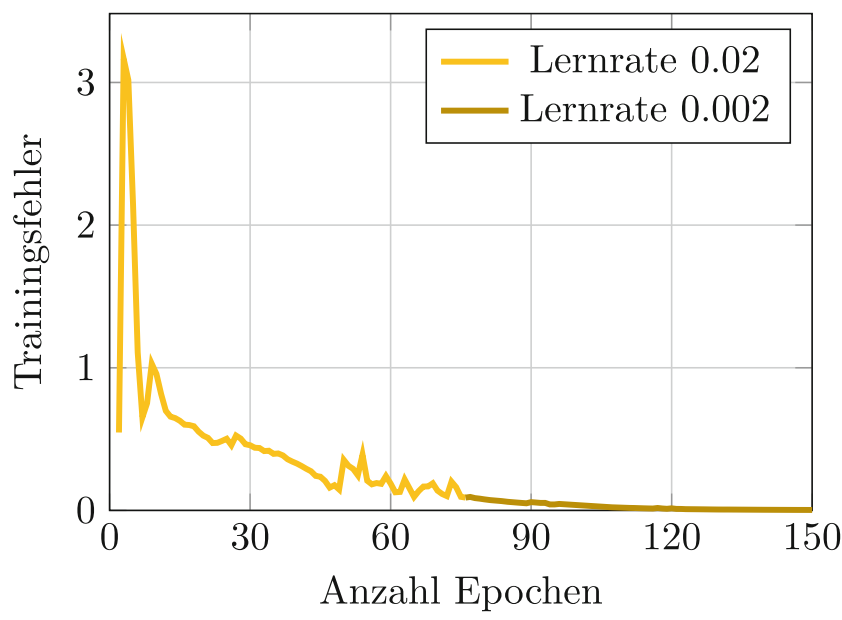

Abb. 10 Wert der Fehlerfunktion beim Training mit zwölf Merkmalen auf den Trainingsdaten von acht Versuchspersonen über die Anzahl der trainierten Epochen nun als Eingang für das vollständig verknüpfte Netzwerk dienen. Dieses fungiert als Klassifikator, sodass es eine Sequenz von Tupeln ausgibt, welche durch die SoftMaxSchicht zu einer Sequenz von Klassifikationsergebnissen verarbeitet wird.

Das Training wird über eine bestimmte Anzahl von 150 Epochen durchgeführt. Diese Anzahl wird so groß gewählt, dass der Fehler nur noch einen kleinen Gradienten besitzt und sich kaum mehr verbessert, wie in Abb. $10 \mathrm{zu}$ sehen ist.

Die initiale Lernrate wird auf 0,02 festgesetzt, welche nach der Hälfte der Epochen noch einmal auf 0,002 verkleinert wird. Dies spiegelt sich im Trainingsfehler in Abb. 10 wider. Hier ist zu erkennen, dass die Varianz des Fehlersignals deutlich abnimmt.

\subsection{Ergebnisse der Fahrerzustandserkennung}

In diesem Abschnitt werden die Ergebnisse der Datenverarbeitung vorgestellt. Es werden verschiedene künstliche neuronale Netzwerke verglichen, die unterschiedlich trainiert sind, aber immer die in Abschn. 5.2 vorgestellte Struktur aufweisen. Der Unterschied liegt darin, dass eine verschiedene Anzahl von Merkmalen aus den drei Merkmalen bei dem Training verwendet wird. Bei jedem Ergebnis wird das Netzwerk auf Daten einer Versuchsperson ausgewertet, die nicht beim Training verwendet wurde. Das Netzwerk ermittelt den Fahrerzustand über die Versuchszeit und anschließend wird das Ergebnis mit dem vorher manuell beschrifteten Wert für den Fahrerzustand (Ground Truth) verglichen. Die Genauigkeit wird in diesem Fall durch die Anzahl der richtig positiven Klassifikationen geteilt durch die Gesamtzahl der Klassifikationen bestimmt, welche im Optimalfall $100 \%$ betragen kann. Hierbei sind hohe Werte der Genauigkeit zu erwarten, denn über $80 \%$ der Zeit bleibt der Zustand in diesem speziellen Fall gleich, sodass ein Netzwerk ohne die Prädiktion eines Zustandswechsel bereits $80 \%$ Genauigkeit erreicht. Als weiteres Maß wird der Zeitunterschied zwischen dem prädizierten Zustandswechsel und dem aus der Beschriftung hervorgehenden $\mathrm{Zu}$ standswechsel verwendet, sodass ein absoluter Vergleichswert vorliegt. Dieser sollte im Optimalfall möglichst gering sein. Dazu werden noch die Werte der Präzision und die Ausfallquote angegeben. Die Präzision bestimmt sich durch die Anzahl der richtig positiven durch die Summe aus richtig positiven und falsch positiven Klassifikationen. Die Ausfallquote bestimmt sich durch die Anzahl der richtig positiven durch die Summe aus richtig positiven und falsch negativen Klassifikationen.

Als Referenz der Genauigkeit ist in Abb. 11 das Ergebnis von einem vollständig verknüpften Netzwerk zu sehen, welches nicht die zeitliche Abfolge der Daten berücksichtigt, da jeder Messpunkt zufällig und von anderen unabhängig für 


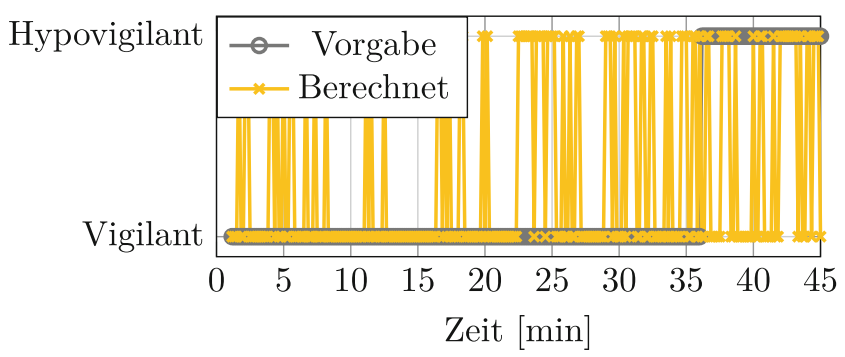

Abb. 11 Klassifikation auf den Testdaten mit allen verfügbaren Merkmalen und einem einfachen Netzwerk

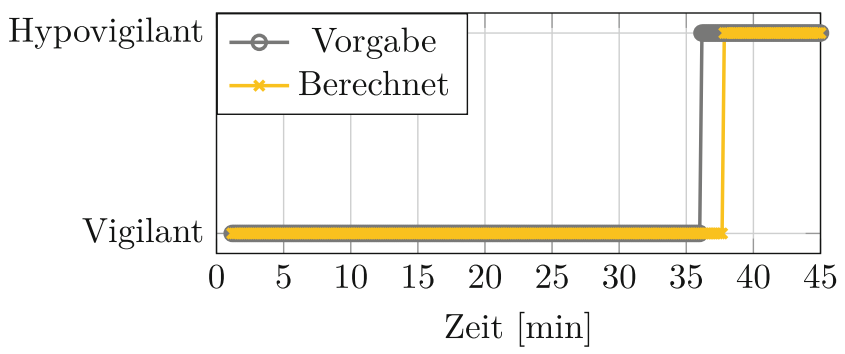

Abb. 12 Klassifikation auf einer Sequenz einer Testdaten mit allen verfügbaren Merkmalen

das Training benutzt wird. Es wird deutlich, dass bei einer Genauigkeit von etwa $65 \%$ viele Messwerte der Sequenz falsch zugeordnet werden und das Ergebnis stark zwischen den Zuständen hin und her springt. Damit ist die Präzision auch nur bei einem Wert von 28,41\% und die Ausfallquote bei einem Wert von 46,30\%. Eine belastbare Aussage lässt sicher daher mit diesem Netz nicht treffen. Gerade die Fehlklassifikationen an sehr späten Zeitpunkten könnten für ein Folgesystem viel relevanter werden und eine Unstetigkeit ist hier nicht erwünscht.

Nachfolgend werden nun die Ergebnisse der Netzwerke mit der Struktur aus Abb. 9 gezeigt und erläutert.

In Abb. 12 ist das Ergebnis des Netzwerks zu sehen, bei dem die Gehirnwellen von dem EEG Stirnband nicht als Merkmale zum Training benutzt werden. Es werden nur die drei verbleiben Merkmale (aus Herz und Auge) ausgewertet. Bei den Testdaten kann eine Genauigkeit von 96\% festgestellt werden. Damit ist das Netz um 31 Prozentpunkte besser als ein einfaches vollständig verknüpftes Netzwerk. Der Zeitverzug zwischen den Zustandswechsel beträgt 100 s. Die Präzision liegt in diesem Fall bei $100 \%$ und die Ausfallquote bei $81,48 \%$. Somit wird er Unterschied zum einfachen Netzwerk deutlich, denn bei diesem Netzwerk mit Gedächtnis springt der Zustand nicht, sodass die Präzision deutlich besser ist. Um die Robustheit des Verfahrens zu testen wird eine Kreuz-Validierung durchgeführt. Dazu werden Test- und Trainingsdaten mehrfach verändert, indem immer eine andere Versuchsperson als Testdatensatz fungiert und die übrigen Acht als Trainingsdatensatz. Dies wird für alle neun Versuchspersonen wiederholt, sodass je-

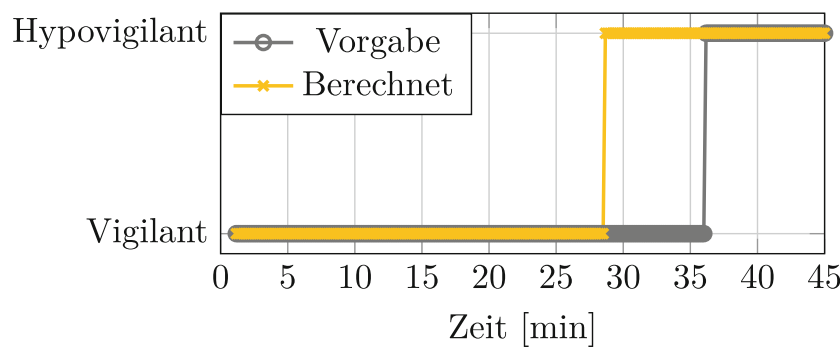

Abb. 13 Klassifikation auf einer Sequenz der Testdaten nur mit PERCLOS als Merkmal

de Versuchsperson einmal als Testdatensatz herangezogen wird. Hierbei erreicht dieses Netzwerk eine Genauigkeit von $\varnothing 86,2 \%$, die sich nur um etwa 10 Prozentpunkte von der Auswertung des einzelnen Testdatensatzes verringert.

Wird das Netzwerk nur mit dem Merkmal der Kameraauswertung (ein Merkmal aus den Augen) trainiert, dann ist das Ergebnis in Abb. 13 zu sehen. Hier kann erkannt werden, dass sich das Ergebnis verschlechtert hat und sich mit etwa $83 \%$ also 13 Prozentpunkte schlechter darstellt. Es wird deutlich, dass das Netzwerk den Wechsel des $\mathrm{Zu}$ stands deutlich zu früh vorhersagt, sodass der Fehler zwischen der Minute 28 und 36 entsteht. Somit lässt sich hier die Zeitdifferenz zwischen den Zustandswechsel mit $450 \mathrm{~s}$ angeben. Diese ist im Vergleich zu dem Netzwerk, das mit mehr Merkmalen trainiert wurde um 90s größer. Die Präzision ist in diesem Fall bei einem Wert von 54,55\% und somit um 45 Prozentpunke schlechter, als bei dem Netzwerk, welches mit Merkmalen aus verschieden Sensoren gespeist wird. Diese Veränderung ergibt sich durch die zu frühe Schätzung, des Zustandwechsels. Die Ausfallquote liegt dahingegen bei $100 \%$ und ist damit um etwa 24 Prozentpunkte besser, als bei dem zuvor gezeigtem Netzwerk. Diese Werte sind stark von dem Testsignal abhängig. Da hier der Zustandswechsel recht spät ist, kann das Netzwerk mit der angegeben Verzugszeit gar nicht besser in dieser Bewertung werden, obwohl erwartet werden kann, dass der Fahrer noch länger in dem veränderten Zustand bleiben würde. Dennoch wird deutlich, dass der berechnete Zustand nicht springt. Dies ist wichtig, da es kritischer wäre eine Fehlklassifikation am Ende der Messung zu erhalten.

Ebenso kann die Auswertung für das Netzwerk durchgeführt werden, welches nur mit den Merkmalen des EKG (also dem Wert der Herzfrequenz $F$ und dem Wert der Herzraten-Variabilität $H$ ) trainiert wird. Das Ergebnis dazu ist in Abb. 14 zu sehen. In diesem Fall wird der Wechsel des Fahrerzustands erst in Minute 40 von dem Netzwerk bestimmt, sodass hier eine Genauigkeit von etwa $90 \%$ resultiert. Dieses Ergebnis ist also sechs Prozentpunkte schlechter als das mit allen Merkmalen trainierte, jedoch sieben Prozentpunkte besser als das Ergebnis, welches nur mit den Merkmalen der Kamera alleine trainiert wurde. Hier ist der zeitliche 


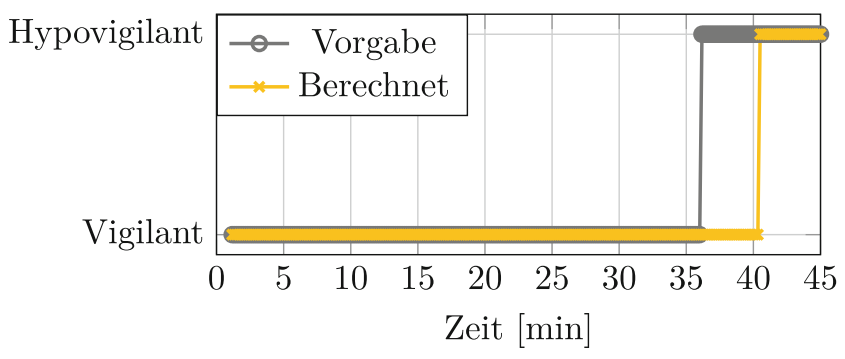

Abb. 14 Klassifikation auf einer Sequenz der Testdaten mit allein Herzdaten als Merkmal

Versatz 270s und somit 70s größer als bei dem Netzwerk, das mit mehr Merkmalen trainiert wurde, jedoch 180 s weniger als bei dem Netzwerk, welches nur mit den Merkmalen aus dem Kamerasensor trainiert wurde. Die Präzision liegt hierbei bei einem Wert von $100 \%$, also genau so hoch, wie bei dem Netzwerk, welches mit allen Merkmalen trainiert wurde und etwa 55 Prozentpunkte besser, als bei dem Netzwerk, welches nur mit Merkmalen vom Herzen arbeitet. Dies lässt sich durch die ebenfalls zu späte Schätzung des Zustandswechsel erklären. So verändert sich auch der Wert der Ausfallquote auf 51,85\%. Dieser Wert ist der schlechteste unter den Netzwerken mit Gedächtnis. Auch hier wird deutlich, dass wie zuvor erwähnt, das Testsignal maßgeblich zu diesem Ergebnis beiträgt. Auch hier ist deutlich, dass das berechnete Signal nach dem Zustandswechsel stetig bleibt. Auch hier ist es wichtig, dass zum Ende das Signal richtig klassifiziert wird, da Fehlklassifikationen an späten Zeitpunkten schlechter wären.

Abschließend kann gesagt werden, dass trotz der fehlenden Aussagekraft der psychologischen Aufgabe eine Veränderung in den kognitiven Signalen am Kopf bei einer längeren automatisierten Fahrt sichtbar wird. Einige Probanden haben nach dem Versuch ihre subjektive Wahrnehmung über den Fragebogen hinaus geschildert. Daraus geht hervor, dass subjektiv die Aufmerksamkeit enorm nachgelassen hat und auch die Augen offen zu halten auf Dauer schwergefallen ist. Ein Proband hat geäußert, dass er versucht hat durch motorische Aktivierung die Aufmerksamkeit hoch zu halten, was sich ebenfalls in den Gehirnwellen widerspiegelt, die sich kaum über die Versuchszeit verändern. Ein anderer Proband schildert ein "schnelles Abschalten", welches auch in den Gehirnwellen durch eine frühe Änderung am Anfang der Versuchszeit deutlich wird. Diese Änderung der Gehirnwellen kann mit einer Kombination aus Augenschlusszeit und Herzmerkmalen mit einem LSTM-Netzwerk mit etwa $96 \%$ Genauigkeit und einer Verzugszeit von 100 s klassifiziert werden. Es kann dabei gezeigt werden, dass die LSTM-Netzwerkstruktur Vorteile gegenüber einfachen vollständig verknüpften Netzwerken bietet und einzelne Sensoren ein schlechteres Klassifikationsergebnis erbringen.

\section{Diskussion und Ausblick}

Mit dieser Arbeit werden zwei Aspekte untersucht: Das Verhalten von Versuchspersonen bei einer längeren automatisierten Autobahnfahrt in einem partiell automatisierten Fahrzeug ohne Übergabesituation und die Erkennung des psychologischen Zustands mit einer Kombination von verschiedenen Sensoren. In der Studie, die mit neun Versuchspersonen durchgeführt wurde, kann gezeigt werden, dass die gewählte Situation für die meisten Versuchspersonen Herausforderungen mit sich bringt. Eine Fahrt mit einem automatisierten System wird als langweilig und ermüdend beschrieben. Somit sind typische Gegebenheiten für eine Unterforderung vorhanden, die zu schlechteren psychologischen Leistungen führen können, wie es auch schon von [19] gezeigt wurde. Einige Versuchspersonen bewerten die Fahrt als gar nicht anstrengend, da durch die Automatisierung der Fahraufgabe keine Aufgaben mehr zu erfüllen sind und zudem keine physische Interaktion für die Fahraufgabe notwendig ist. Die anderen Versuchspersonen scheinen die psychische Anstrengung des aufmerksam Bleibens zu bewerten, welche eher hoch eingeschätzt wird. Dies zeigt, dass eine hohe Automatisierung (aber immer noch partiell nach SAE Level 2, wie oben beschrieben) bei längeren monotonen Fahrten möglicherweise keinen Komfortzugewinn mit sich bringt. In diesem Zusammenhang könnte eine dynamische Aufgabenverteilung zwischen Fahrer und Fahrzeug untersucht werden, um herauszufinden, ob der Fahrer in dem Fall, das er zeitweilig in die Fahraufgabe mit integriert wird, weniger Langeweile erfährt. So könnte der Fahrer vigilant gehalten und kontinuierlich beobachtet werden, ob er diesen aktiven Status beibehält.

Bei den Messungen der Reaktionszeiten zeigt sich, dass die Personen auch nach einer längeren Zeit als 30 Minuten noch fähig sind, auf seltene Straßenschilder zu reagieren. Somit lässt sich festhalten, dass die Vigilanz bei der hier vorgestellten Signalentdeckungsaufgabe nicht deutlich durch Reaktionszeiten messbar gemacht werden konnte. Die Reize durch die Straßenschilder sind so groß, dass selbst nach 90Minuten noch auf diese reagiert werden kann. Nur wenige Versuchspersonen zeigen vereinzelt ausbleibende Reaktionen auf Straßenschilder. Die Reize für die Versuchsperson müssten noch schwächer sein, um einen solchen Effekt zu sehen. Auch Kommentare der Versuchspersonen nach dem Versuch sagen aus, dass ein kommendes Schild schnell und einfach erkannt werden kann. Dennoch ist die hier vorgestellte automatisierte Fahrt sehr ermüdend und kann Einfluss auf die Vigilanz haben, so wird dieser Effekt durch eine starke Änderung in den Gehirnwellen sichtbar. Die Gehirnwellen sind, neben den Reaktionszeiten, am besten für die Erfassung der kognitiven Eigenschaften der Versuchspersonen geeignet, wie für den Zustand der Müdigkeit schon von [2] genauer unter- 
sucht wurde. So kann mit dieser Messmethode ein Wechsel in dem kognitiven Fahrerzustand festgestellt werden, wie es auch von [19] zusammengefasst wurde. Zukünftige Studien sollten somit mit schwächeren Reizen durchgeführt werden, sodass der Effekt der Vigilanz tatsächlich bei mehreren Probanden in den Reaktionszeiten sichtbar wird. Besonders die Prüfung des fahrsicheren Zustands ist in einem partiell automatisierten Fahrzeug noch immer eine Aufgabe, die der Fahrer übernehmen muss. So könnte eine Aufgabe zur Sicherstellung des fahrsicheren Zustand die Geschwindigkeitsüberprüfung darstellen.

Die Verarbeitung mit den künstlichen neuronalen Netzwerken hat gezeigt, dass es möglich ist, mit den verschiedenen Merkmalen einen Wechsel des Fahrerzustands zu berechnen. In dieser Arbeit kann gezeigt werden, dass durch die Fusion von mehreren Sensoren ein besseres Ergebnis bei der Erkennung des Fahrerzustands erreicht werden kann. Dabei sind die Gehirnwellen, die zum Einordnen der Daten verwendet werden, nicht als Merkmal für ein gutes Ergebnis der Klassifikation notwendig. Die Merkmale aus dem Auge und von dem Herzen enthalten vermutlich ähnliche Informationen, wie die visuell direkt in den Gehirnwellen sichtbaren. Da die Generalisierbarkeit des Modells durch die geringe Zahl an Probanden nicht beurteilt werden kann, wird die Robustheit von dem Modell, welches die besten Ergebnissen in der Genauigkeit zeigt, durch eine KreuzValidierung gezeigt. So kann davon ausgegangen werden, dass auch bei großen Unterschieden zwischen den einzelnen Versuchspersonen ein Netzwerk trainiert werden kann. Die Verzugszeit von etwa 100 s ist im Vergleich zu den langen Zeiträumen in denen verminderte Vigilanz auftreten kann (mehr als $30 \mathrm{~min}$ ) eher gering. Durch die kleine Stichprobe in dieser Machbarkeitsstudie können diese Zusammenhänge noch nicht generalsiert werden, es wird nur eine Tendenz zur Machbarkeit gezeigt. Um diese Ansätze besser generalisieren zu können, sollte in einer weiteren Studie mit mehr Versuchspersonen gearbeitet werden.

Die Messung des Herzens findet in dem vorliegenden Fall noch kontaktbehaftet statt, jedoch gibt es in der Forschung schon Ansätze diese Messung kontaktlos durchzuführen. So wird in [24] ein System in einem Autositz entwickelt, welches mit einem Radarsensor die einzelnen Herzschläge von sitzenden Personen erfassen kann. Ein anderer Ansatz wird in [30] vorgestellt, bei dem das Bild einer Farbkamera so gefiltert wird, dass auch hier einzelne Herzschläge sichtbar gemacht werden können. Mit diesen Ansätzen könnte in Zukunft die Herzfrequenz $F$ und der Wert der Herzraten-Variabilität $H$ kontaktlos erfasst werden. Bei der Auswertung des Netzwerks, welches nur die Merkmale der Kamera verwendet hat, ist gezeigt worden, dass der Fahrerzustand zu früh errechnet wird. Zudem ist noch nicht berücksichtigt, dass durch Kontaktlinsen die Augenschließzeit verändert werden könnte. Auch dieser Zusammenhang muss in zukünftigen Untersuchungen berücksichtigt werden.

Die Hyperparameter für die Struktur der neuronalen Netze und für das Training werden in dieser Arbeit immer gleich gehalten, um einen Vergleich zu ermöglichen. Wenn in Zukunft eine Sensorkonfiguration festgehalten wird, ist es sinnvoll, für diese dann das neuronale Netzwerk weiter zu optimieren. Dabei sollte ein größerer Datensatz verwendet werden, um mehr individuelle Unterschiede abdecken zu können. Bei dem Training der Netze kann noch mit der Anzahl der Epochen experimentiert werden, um sowohl eine hohe Modellgüte, als auch eine geringere Überanpassung zu erreichen. Ebenso dienen in dieser Arbeit nur Sequenzen von 45 Minuten als Eingangssignal. Ein Wechsel des Fahrerzustands geschieht jedoch in kürzerer Zeit. In Zukunft sind verschiedene Sequenzlängen zu untersuchen, sodass die Sequenz wie ein Fenster über das Signal geschoben wird und das neuronale Netzwerk kürzere Prognosen, aber dafür in kürzeren Zeitabständen abgeben könnte.

Zudem könnten noch weitere Merkmale für die Fahrerzustandserkennung herangezogen werden. Der motorische Zustand kann mit dem kognitiven Zustand verbunden sein und so weitere Erkenntnisse bei der Beobachtung des Fahrers erbringen. Dazu ist bereits ein Sitzdrucksensorsystem mit zwölf Drucksensoren in dem Fahrersitz implementiert. Diese Daten sollen in Zukunft in das Sensorsetup für die nächsten Untersuchungen mit aufgenommen werden, um so weitere Informationen über den Fahrer in die Berechnung einfließen zu lassen. Die resultierenden Merkmale sollten dann ebenfalls noch ähnlich wie in dem vorliegenden Beitrag überprüft werden, um die Relevanz dieser Merkmale herauszustellen.

Funding Open Access funding enabled and organized by Projekt DEAL.

Interessenkonflikt A. Hugenroth, A. Warnecke und T. Bertram geben an, dass kein Interessenkonflikt besteht.

Open Access Dieser Artikel wird unter der Creative Commons Namensnennung 4.0 International Lizenz veröffentlicht, welche die Nutzung, Vervielfältigung, Bearbeitung, Verbreitung und Wiedergabe in jeglichem Medium und Format erlaubt, sofern Sie den/die ursprünglichen Autor(en) und die Quelle ordnungsgemäß nennen, einen Link zur Creative Commons Lizenz beifügen und angeben, ob Änderungen vorgenommen wurden.

Die in diesem Artikel enthaltenen Bilder und sonstiges Drittmaterial unterliegen ebenfalls der genannten Creative Commons Lizenz, sofern sich aus der Abbildungslegende nichts anderes ergibt. Sofern das betreffende Material nicht unter der genannten Creative Commons Lizenz steht und die betreffende Handlung nicht nach gesetzlichen Vorschriften erlaubt ist, ist für die oben aufgeführten Weiterverwendungen des Materials die Einwilligung des jeweiligen Rechteinhabers einzuholen.

Weitere Details zur Lizenz entnehmen Sie bitte der Lizenzinformation auf http://creativecommons.org/licenses/by/4.0/deed.de. 


\section{Literatur}

1. Åkerstedt T, Gillberg M (1990) Subjective and objective sleepiness in the active individual. Int J Neurosci 52(1-2):29-37. https://doi. org/10.3109/00207459008994241

2. Coetzer RC, Hancke GP (2009) Driver fatigue detection: a survey. In: IEEE (Hrsg) AFRICON 2009, S 1-6 https://doi.org/10.1109/ AFRCON.2009.5308101

3. Daimler AG ATTENTION ASSIST: Müdigkeitserkennung warnt rechtzeitig vor dem gefährlichen Sekundenschlaf. https://web. archive.org/web/20160304111653/ (Erstellt: 12. Nov. 2008). Zugegriffen: 3. Nov. 2020 (http://media.daimler.com/dcmedia/0-921658892-49-1147698-1-0-0-1147922-0-1-11694-0-0-1-0-0-0-0$0 . \mathrm{html}$ ?TS=1361030865949)

4. Europäische Kommission Sicherheit im Straßenverkehr: Kommission begrüßt Einigung auf neue EU-Vorschriften, die zur Rettung von Menschenleben beitragen. https://ec.europa.eu/commission/ presscorner/detail/de/IP_19_1793 (Erstellt: 26. März 2019). Zugegriffen: 28. Okt. 2020

5. Feldhütter A, Feierle A, Kalb L, Bengler K (2018) A new approach for a real-time non-invasive fatigue assessment system for automated driving. Proc Hum Factors Ergon Soc Annu Meet 62:1669-1673. https://doi.org/10.1177/1541931218621379

6. Gilgen-Ammann R, Schweizer T, Wyss T (2019) Rr interval signal quality of a heart rate monitor and an ecg holter at rest and during exercise. Eur J Appl Physiol 119(7):1525-1532. https://doi.org/10. 1007/s00421-019-04142-5

7. Haarmann A (2007) Der einsatz psychophysiologischer variablen bei der adaptiven automatisierung am beispiel einer flugsimulationsaufgabe. Dissertation, Bergische Universität Wuppertal, Wuppertal, http://nbn-resolving.de/urn/resolver.pl?urn=urn3Anbn3Ade 3Ahbz3A468-20070925

8. Hecht T, Feldhütter A, Radlmayr J, Nakano Y, Miki Y, Henle C, Bengler K (2019) A review of driver state monitoring systems in the context of automated driving. In: Advances in intelligent systems and computing Proceedings of the 20th Congress of the International Ergonomics Association (IEA 2018). Bd. 823. Springer, Cham, S 398-408 https://doi.org/10.1007/978-3-319-96074-6_43

9. Hochreiter S, Schmidhuber J (1997) Long short-term memory. Neural Comput 9(8):1735-1780

10. Interaxon (2015) MuseIO I Available data. https://web.archive. org/web/20181105231756/. Zugegriffen: 3. Nov. 2020 (http:// developer.choosemuse.com/tools/available-data\#Absolute_Band_ Powers)

11. Jürgensohn T, Böhm S, Gardas D, Stephani T (2018) Entwicklung der Fahr- und Verkehrskompetenz mit zunehmender Fahrerfahrung. Berichte der Bundesanstalt für Strassenwesen Mensch und Sicherheit. Carl Ed. Schünemann KG, Bremen

12. Kalsbeek J, Sykes RN (1967) Objective measurement of mental load. Acta Psychol 27:253-261. https://doi.org/10.1016/00016918(67)90066-2

13. Krajewski J, Sommer D, Trutschel U, Edwards D, Golz M (2009) Steering wheel behavior based estimation of fatigue. In: Proceedings of the Fifth International Driving Symposium on Human Factors in Driver Assessment, S 118-124 https://doi.org/10.17077/ drivingassessment.1311

14. Kundinger T, Wintersberger P, Riener A (2019) (Over)trust in automated driving. In: Brewster S, Fitzpatrick G, Cox A, Kostakos V (Hrsg) Extended Abstracts of the 2019 CHI Conference on Human Factors in Computing Systems. ACM, New York, S 1-6 https://doi. org/10.1145/3290607.3312869 ((05022019))

15. Leonhardt S, Leicht L, Teichmann D (2018) Unobtrusive vital sign monitoring in automotive environments-A review. Sensors. https://doi.org/10.3390/s18093080
16. Liu H, Hiraoka T (2019) Driving behavior model considering driver's over-trust in driving automation system. In: Janssen CP, Donker SF, Chuang LL, Ju W (Hrsg) Proceedings of the 11th International Conference on Automotive User Interfaces and Interactive Vehicular Applications: Adjunct Proceedings. ACM, New York, S 115-119 https://doi.org/10.1145/3349263.3351525 ((09212019))

17. Mackworth NH (1948) The breakdown of vigilance during prolonged visual search. Q J Exp Psychol. https://doi.org/10.1080/ 17470214808416738

18. Marquart G, Cabrall C, de Winter J (2015) Review of eye-related measures of drivers' mental workload. Procedia Manuf 3:2854-2861. https://doi.org/10.1016/j.promfg.2015.07.783

19. McWilliams T, Ward N (2021) Underload on the road: Measuring vigilance decrements during partially automated driving. Front Psychol 12:631,364. https://doi.org/10.3389/fpsyg.2021.631364

20. (2019) Chapter five - Biomechatronic applications of brain-computer interfaces. In: Novak D (Hrsg) Handbook of biomechatronics. Elsevier, Amsterdam https://doi.org/10.1016/B978-0-12-8125397.00008-8

21. Ogino M, Mitsukura Y (2018) Portable drowsiness detection through use of a prefrontal single-channel electroencephalogram. Sensors. https://doi.org/10.3390/s18124477

22. O'Kane S Tesla rejected more advanced driver monitoring features on its cars. https://www.theverge.com/2018/5/14/17352814/elonmusk-tesla-autopilot-face-tracking-gm (Erstellt: 14. Mai 2018). Zugegriffen: 6. Nov. 2020

23. Sahayadhas A, Sundaraj K, Murugappan M (2012) Detecting driver drowsiness based on sensors: a review. Sensors 12(12):16,93716,953

24. Schires E, Georgiou P, Lande TS (2018) Vital sign monitoring through the back using an uwb impulse radar with body coupled antennas. IEEE Trans Biomed Circuits Syst 12(2):292-302

25. Shaffer F, Ginsberg JP (2017) An overview of heart rate variability metrics and norms. Front Public Health. https://doi.org/10.3389/ fpubh.2017.00258

26. Sommer D, Golz M (2010) Evaluation of perclos based current fatigue monitoring technologies. In: 2010 Annual International Conference of the IEEE Engineering in Medicine and Biology. IEEE, S $4456-4459$

27. Ford Motor Company $\mathrm{T}$ Ford Co-Pilot $360^{\mathrm{TM}}$ technology adds hands-free driving, over-the-air updates and more to help ford customers feel more relaxed and confident. https://media.ford.com/ content/fordmedia/fna/us/en/news/2020/06/18/ford-co-pilot360technology-adds-hands-free-driving.html (Erstellt: 18. Juni 2020). Zugegriffen: 28. Okt. 2020

28. Vicente F, Huang Z, Xiong X, de La Torre F, Zhang W, Levi D (2015) Driver gaze tracking and eyes off the road detection system. IEEE Trans Intell Transport Syst 16(4):2014-2027

29. Wierwille WW, Ellsworth LA (1994) Evaluation of driver drowsiness by trained raters. Accid Anal Prev 26(5):571-581

30. Wu HY, Rubinstein M, Shih E, Guttag J, Durand F, Freeman W (2012) Eulerian video magnification for revealing subtle changes in the world. ACM Trans Graph 31(5):1-8

31. Xiao Z, Hu Z, Geng L, Zhang F, Wu J, Li Y (2019) Fatigue driving recognition network: fatigue driving recognition via convolutional neural network and long short-term memory units. IET Intell Transp Syst 13(9):1410-1416. https://doi.org/10.1049/iet-its.2018. 5392

32. Cao Z, Hidalgo Martinez G, Simon T, Wei S, Sheikh YA (2019) Openpose: Realtime multi-person $2 \mathrm{~d}$ pose estimation using part affinity fields. IEEE Trans Pattern Anal Mach Intell 43(1):172-186. https://doi.org/10.1109/TPAMI.2019.2929257

33. Zhang X, Li J, Liu Y, Zhang Z, Wang Z, Luo D, Zhou X, Zhu M, Salman W, Hu G et al (2017) Design of a fatigue detection system for high-speed trains based on driver vigilance using a wi- 
reless wearable eeg. Sensors 17(3):486. https://doi.org/10.3390/ s17030486

34. Zschocke S (2002) Klinische Elektroenzephalographie. Springer eBook Collection Medicine. Springer, Berlin, Heidelberg https:// doi.org/10.1007/978-3-662-08106-8 